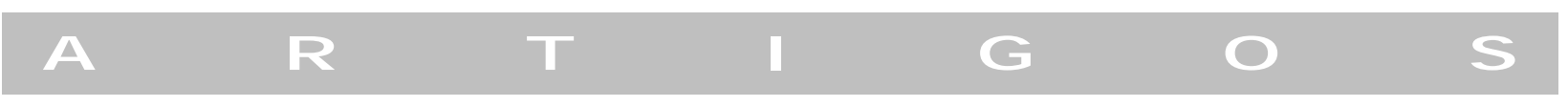

\title{
A estrutura e o conteúdo universais dos valores humanos: análise fatorial confirmatória da tipologia de Schwartz
}

\author{
Valdiney V. Gouveia \\ Universidade Federal da Paraíba \\ Eva Martínez \\ Universidad Complutense de Madrid \\ Maja Meira \\ Taciano Lemos Milfont \\ Universidade Federal da Paraíba
}

\begin{abstract}
Resumo
O presente estudo teve como objetivos (1) comprovar a existência dos dez tipos motivacionais de valores e (2) avaliar em que medida estes mantém relações de compatibilidades e conflitos entre si, segundo a teoria de Schwartz (1992). Participaram 477 estudantes universitários, a maioria mulher (77\%) e não-religiosa (78\%), com uma média de idade de 21 anos (amplitude de 18 a 49 anos). Estes responderam ao Questionário de Valores de Schwartz e a um conjunto de perguntas demográficas. Uma análise fatorial confirmatória (LISREL) indicou a adequação da tipologia dos dez tipos de valores $\left(G F I=0,87\right.$, razão $\left.\chi^{2} / g l=3,81\right)$. Em relação a estrutura de compatibilidades e conflitos entre os valores, esta foi plenamente confirmada no primeiro caso e parcialmente no segundo. Concluiu-se que a teoria em questão é adequada; a não comprovação de alguns conflitos entre os tipos motivacionais pode se dever à complexidade da dinâmica dos valores, não apreendida através dos modelos de equações estruturais.

Palavras-chave: Valores, Tipos Motivacionais, LISREL, Schwartz.
\end{abstract}

\begin{abstract}
The universals structure and content of human values: Confirmatory factor analysis of Schwartz's typology. The present study aimed (1) to check the existence of the ten motivational types of values, and (2) to evaluate in which ways these values maintain relationships of compatibilities and conflicts to each other, according to the Schwartz's theory (1992). The participants were 477 undergraduate students, most women (77\%) and not religious (78\%), with an average age of 21 years (ranging from 18 to 49). They answered Schwartz Values Survey and a set of demographic questions. A factor confirmatory analysis (LISREL) indicated the appropriateness of the typology of the ten types of values $\left(\mathrm{GFI}=.87\right.$, ratio $\left.\chi^{2} / \mathrm{gl}=3.81\right)$. In relation to the structure of compatibilities and conflicts among the values, this was confirmed fully in the first case and confirmed partially in the second one. It was concluded that Schwartz's theory presents an adequate fit to the data; the non confirmation of some conflicts among the motivational types can be due to the complexity of the dynamics of the human values, not apprehended through the structural equation models.
\end{abstract}

Key words: Values, Motivational Types, LISREL, Schwartz.

$\mathrm{E}$ mbora tenha raízes no início do século passado (Thomas \& Znaniecki, 1918), o tema dos valores humanos em Psicologia Social se constituiu objeto de pesquisa científica principalmente nas três últimas déca- das. Milton Rokeach, com a publicação do seu livro The nature of human values (Rokeach, 1973), conseguiu ao menos quatro grandes feitos: (1) propôs uma abordagem que reuniu aspirações de diversas áreas, como a Antropologia, a 
Filosofia, a Sociologia e, por suposto, a Psicologia; (2) diferenciou os valores de outros construtos com os quais costumavam ser relacionados, como as atitudes, os interesses e os traços de personalidade; (3) apresentou um instrumento que, pela primeira vez, tratava de medir os valores como um construto legítimo e específico; e (4) demonstrou sua centralidade no sistema cognitivo das pessoas, reunindo dados sobre seus antecedentes e conseqüentes. Seus estudos tiveram impacto também no Brasil, onde seu instrumento foi traduzido (Günther, 1981) e empregado para conhecer, por exemplo, em que medida os valores estariam relacionados com o sexo e a idade das pessoas (Tamayo, 1988).

Muitos foram os pesquisadores que seguiram Rokeach (Braithwaite \& Law, 1985; Feather, 1984; Schwartz \& Bilsky, 1987). Não obstante, três aspectos são geralmente mencionados como problemáticos em sua abordagem (Gouveia, 1998; Molpeceres, 1994; Tamayo, 1997): (a) o tipo de medida empregada, que é de natureza ipsativa (sugere uma dependência entre as pontuações de um mesmo sujeito); (b) a indefinição da estrutura dos valores; e (c) a restrição das amostras dos seus estudos, realizados principalmente com estudantes universitários norte-americanos. Frente a estas limitações, outros modelos teóricos têm sido propostos (Gouveia, 1998), entre os quais se destaca o de Shalom H. Schwartz e seus colaboradores (Schwartz \& Bilsky, 1987, 1990; Tamayo \& Schwartz, 1993; Grad, Ros, Álvaro \& Torregrosa, 1993).

O modelo teórico de Schwartz é claramente uma extensão daquele que propôs Rokeach, com três diferenças principais: (a) a proposta de uma medida que combina intervalos com âncoras (geralmente dois valores, um avaliado como de máxima importância e outro que é identificado como contrário aos demais valores do respondente); (b) a ênfase na base motivacional como explicação para a estrutura dos valores; e (c) a sugestão da universalidade da estrutura e do conteúdo dos tipos motivacionais de valores. Este modelo tem reunido dados bastante consistentes que, em grande medida, o suportam trans-culturalmente (Schwartz \& Sagiv, 1995). Análises realizadas dentro das culturas são também favoráveis, como se constatou no Brasil (Tamayo, 1994; Tamayo \& Schwartz, 1993) e em outros países com os quais mantém um padrão cultural próximo, como Espanha (Ros \& Grad, 1991; Vera \& Martínez, 1994), México (Bilsky \& Peters, 1999) e Portugal (Menezes \& Campos, 1997).

Apesar das evidências empíricas acumuladas, este modelo tem recebido críticas (Gouveia, 1998). Uma das principais diz respeito ao procedimento estatístico empregado para corroborá-lo, a Análise do Menor Espaço (Smallest Space Analysis, SSA), que se baseia no escalonamento multidimensional. Por exemplo, quando um método de Análise Fatorial é empregado para tratar os dados (correlações entre o conjunto de valores específicos), tem sido observada uma estrutura mais complexa (Gendre \& Dupont, 1992; Karp, 1996) do que a relatada por Schwartz $(1992,1994)$. Tamayo (1997) reconhece esta possibilidade e sugere uma abordagem estatística alternativa para dirimir dúvidas a respeito:

Os resultados obtidos através destas análises [SSA] estão na base da elaboração da teoria e da própria validação do Inventário de Valores [de Schwartz]. Acontece que a SSA situa os valores no espaço multidimensional, mas não fornece indicações estatísticas definitivas sobre a orientação e localização precisa dos eixos que organizam os diversos grupos de valores [tipos motivacionais]. Desta forma, no momento de traçar os eixos o pesquisador pode seguir, dentro dos limites já definidos pelo SSA, o caminho mais favorável para a verificação da teoria. Do meu ponto de vista atual, o uso do SSA já deu a sua preciosa contribuição. Talvez seja o momento de pensar em análises confirmatórias para verificar de forma mais objetiva a consistência dos tipos motivacionais de valores e de utilizar modelos LISREL para estabelecer as relações dinâmicas entre eles (p. 130).

O que Tamayo (1997) ressalta é a indeterminação estatística quando a técnica de SSA é empregada. Os mais comuns indicadores de bondade de ajuste desta técnica estatística, o RSQ e o $S$-Stress, referem-se à dimensionalidade do espaço onde serão representadas as distâncias (correlações) entre os itens e/ou valores. Neste sentido, definida a representação espacial, o pesquisador pode traçar uma linha ou desenhar uma figura que reuna os itens que supostamente pertencem ao "fator" que teorizou, existindo portanto pouca possibilidade de que se refute seu modelo. A técnica de análise fatorial convencional ou exploratória é mais criteriosa neste aspecto, fornecendo diversos indicadores sobre a estrutura do conjunto de itens (Bisquerra, 1989): (1) o KMO e o Teste de Esfericidade de Bartlett permitem decidir sobre a adequação de se realizar esta análise estatística; (2) o eigenvalue igual ou superior a 1,00 e sua representação gráfica (o scree test) possibilitam identificar o número de fatores a extrair; e (3) a magnitude da carga fatorial, geralmente acima de 0,30 , sugere a adequação do item como uma representação comportamental de um determinado fator. Esta última técnica estatística, não obstante, não permite estabelecer a priorie comprovar uma estrutura teórica definida; 0 que fornece são indicações sobre como os itens podem ser reunidos em fatores, segundo as respostas de um grupo de participantes. Neste contexto, surge a proposta da análise fatorial confirmatória, que se baseia nos modelos de equações estruturais mencionados previamente. As limitações das duas técnicas estatísticas antes citadas são superadas com esta última, que fornece vários indicadores de bondade de ajuste da teoria em relação aos dados empíricos (Bollen, 1989; Byrne, 1989; Joreskög \& Sörbom, 1989). 
Esta situação animou a realização do presente estudo, cujos objetivos principais foram: (1) comprovar a existência dos dez tipos motivacionais de valores e (2) avaliar em que medida estes mantêm relações de compatibilidades e conflitos entre si, de acordo com o que estabelece a teoria de Schwartz (1992) no nível individual de análise. Para tanto, realizar-se-á uma análise fatorial confirmatória com um conjunto de itens que operacionaliza os tipos motivacionais de valores. Uma vez que esta teoria é conhecida no Brasil (Tamayo, 1994, 1997; Tamayo \& Schwartz, 1993), procurar-se-á resumi-la a seguir.

\section{A Teoria do Conteúdo e da Estrutura Universais dos Valores}

S. H. Schwartz tem o grande mérito de conseguir reunir em torno de sua teoria dos valores pesquisadores dos cinco continentes, em mais de 50 países. Desde o começo do seu modelo (Schwartz \& Bilsky, 1987, 1990), este autor manteve-se fiel à sua idéia principal de que existe um conjunto de motivações universais que dão origem e organizam os diversos valores nas diferentes culturas. Sua teoria define um valor como um conceito ou crença do indivíduo sobre uma meta (terminal, instrumental) que transcende às situações e expressem interesses (individualistas, coletivistas ou mistos) correspondentes a um domínio motivacional (hedonismo, poder, tradição, universalismo etc.), sendo avaliada em uma escala de importância (nada importante, muito importante) como um princípio que guia a sua vida (Schwartz \& Bilsky, 1987).

Excetuando o atributo "escala de importância", que representa uma opção do autor, os demais compreendem facetas que Schwartz e seus colaboradores procuram comprovar empiricamente. No caso das metas, embora inicialmente tenha se argumentado a seu favor (Schwartz \& Bilsky, 1987), logo se concluiu que expressavam principalmente uma questão de semântica, e que valores terminais poderiam ser entendidos como instrumentais e vice-versa (Schwartz, 1992). O tipo de interesse a que servem os valores tem sido objeto de diversas pesquisas, sendo os resultados no geral consistentes com o modelo teórico (Oishi, Schimmack, Diener \& Suh, 1998; Ros \& Gómez, 1997; Schwartz, 1990). Quanto aos tipos motivacionais, compreendem o ponto central da sua teoria e em torno dos quais Tamayo (1997) elabora a crítica antes descrita. Schwartz $(1992,1994)$ não supõe que os valores humanos individualmente sejam universais, senão que seriam universais as motivações que os sustentam. Estas dariam origem aos tipos de valores ou tipos motivacionais, os quais são tratados em termos do seu conteúdo e da sua relação dinâmica de compatibilidade e conflito entre si.

\section{Conteúdo dos Tipos Motivacionais}

São dez os tipos motivacionais de valores, a saber: autodireção, estimulação, hedonismo, realização, poder, benevolência, conformidade, tradição, segurança e universalismo. Cada um destes dá origem a um grupo de valores que, por se correlacionarem diretamente entre si, formariam uma região em uma representação espacial ou propriamente um fator, no caso de uma análise fatorial. $\mathrm{Na}$ Tabela 1, é definido cada tipo de valor com seus valores específicos mais representativos.

Tabela 1.

Definição dos tipos motivacionais de valores universais (com exemplos de valores específicos entre parênteses)

\begin{tabular}{|c|c|}
\hline Autodireção (AD) & Independência no pensamento e na tomada de decisão, criação e exploração (criatividade, independente, liberdade). \\
\hline Estimulação (ES) & Ter excitação, novidade e mudança na vida (ser atrevido, uma vida excitante, uma vida variada). \\
\hline Hedonismo (HE) & Prazer ou gratificação sensual para a própria pessoa (desfrutar da vida, prazer). \\
\hline Realização (RE) & Êxito pessoal como resultado da demonstração de competência segundo as normas sociais (ambicioso, capaz, obter êxito). \\
\hline Poder (PO) & Posição e prestígio social, controle ou domínio sobre pessoas e recursos (autoridade, poder social, riqueza). \\
\hline Benevolência (BE) & $\begin{array}{l}\text { Preservar e reforçar o bem-estar das pessoas próximas com quem se tem um contato pessoal freqüente e não casual (ajudando, } \\
\text { honesto, não rancoroso, ter sentido na vida). }\end{array}$ \\
\hline Conformidade $(\mathbf{C O})$ & $\begin{array}{l}\text { Limitar as ações, inclinações e impulsos que possam prejudicar a outros e violar expectativas ou normas sociais (auto- } \\
\text { disciplina, bons modos, obediência). }\end{array}$ \\
\hline Tradição (TR) & $\begin{array}{l}\text { Respeitar, comprometer-se e aceitar os costumes e as idéias que a cultura tradicional ou a religião impõem à pessoa (devoto, } \\
\text { honra aos pais e mais velhos, humilde, respeito pela tradição, vida espiritual). }\end{array}$ \\
\hline Segurança (SE) & $\begin{array}{l}\text { Conseguir segurança, harmonia e estabilidade na sociedade, nas relações interpessoais e na própria pessoa (ordem social, } \\
\text { segurança familiar, segurança nacional). }\end{array}$ \\
\hline Universalismo (UN) & $\begin{array}{l}\text { Compreensão, apreço, tolerância e proteção em direção ao bem-estar de toda a gente e da natureza (aberto, amizade } \\
\text { verdadeira, igualdade, justiça social, protetor do meio ambiente, sabedoria, um mundo em paz, um mundo de beleza). }\end{array}$ \\
\hline
\end{tabular}


Estes tipos de valores são derivados de três requerimentos humanos universais (Schwartz \& Bilsky, 1987, 1990): as necessidades básicas (organismo), os motivos sociais (interação) e as demandas institucionais para o bem-estar e a sobrevivência dos grupos. Em função de quem se beneficia quando a pessoa adota ou se comporta de acordo com cada tipo de valor, estes autores definem os diferentes interesses que podem cumprir: individualista (poder, realização, hedonismo, estimulação e autodireção), coletivista (tradição, conformidade e benevolência) ou misto (segurança e universalismo). Uma outra forma de considerar os tipos de valores é em razão das suas dimensões de ordem superior, a saber (Schwartz, 1994): auto-promoção vs. autotranscendência e abertura à mudança vs. conservação (ver Figura 1). Não é o propósito do presente estudo considerar as dimensões de interesse ou de ordem superior correspondentes aos tipos de valores; interessa unicamente comprovar a existência destes enquanto estruturas latentes de primeira ordem, por isso serão tratados com mais detalhe a seguir.

\section{Estrutura de Compatibilidade e Conflito dos Tipos Motivacionais}

Segundo Sagiv e Schwartz (1995), "as ações empreendidas na consecução de um tipo de valor têm consequiências psicológicas, práticas e sociais que podem ser conflituosas ou compatíveis com a perseguição de um outro tipo de valor" (p. 438). Como apresentados na Figura 1, os tipos de valores são organizados em uma estrutura circular, significando que aqueles adjacentes são compatíveis e os localizados em extremos opostos representam conflitos.

Schwartz (1992), considerando participantes de 20 países, classificados em 40 amostras, procurou demonstrar a universalidade das compatibilidades e dos conflitos dos tipos de valores. Concluiu, através da técnica SSA, que estes são claramente identificados em ao menos $70 \%$ das amostras. Os seguintes pares de tipos de valores são estimados como compatíveis: poder-realização, realização-hedonismo, hedonismo-estimulação, estimulação-autodireção, autodireção-universalismo, universalismo-benevolência, benevolência-conformidade, conformidade-tradição, tradição-segurança, segurança-poder e segurança-conformidade. Os conflitos esperados são: autodireção-conformidade, autodireção-tradição, autodireção-segurança, estimulaçãoconformidade, estimulação-tradição, estimulação-segurança, universalismo-poder, universalismo-realização, benevolência-poder, benevolência-realização, hedonismo-conformidade e hedonismo-tradição.

Considerando o propósito de comprovar a existência dos dez tipos motivacionais de valores e a adequação da estrutu-

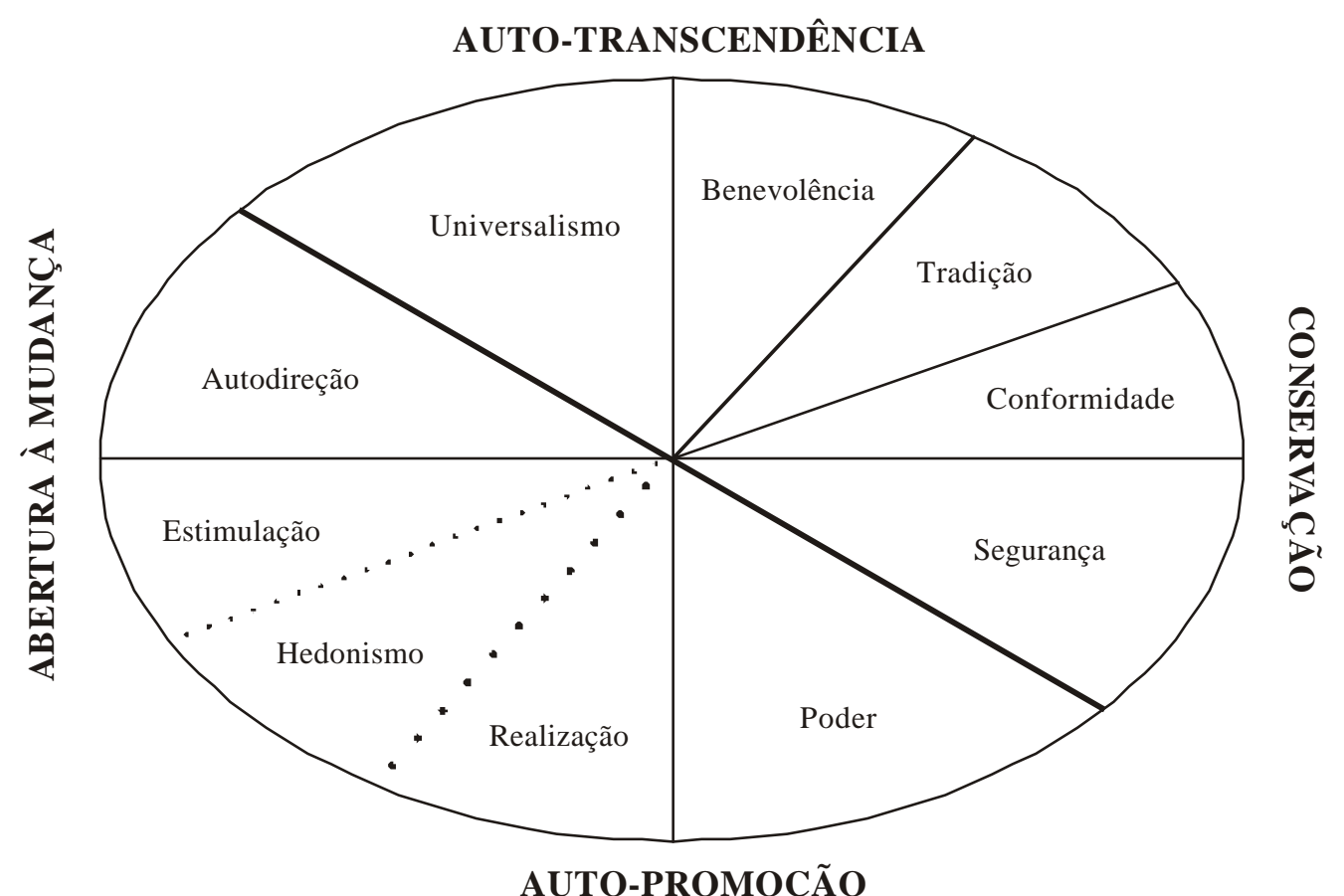

Figura 1. Estrutura Bidimensional dos Tipos Motivacionais (adaptado de Schwartz, 1991, p. 14) 
ra de compatibilidades e conflitos entre estes, pensou-se na realização do estudo descrito a seguir.

\section{Método}

\section{Amostra}

Participaram da presente pesquisa 477 estudantes universitários, sendo 173 alunos de Psicologia e 304 de Sociologia de uma universidade pública da Espanha. A idade média destes foi de 21 anos, tendo o mais jovem 18 e o mais velho 49 anos. A maioria era mulher (77\%), assumindo não ser religiosa $(78 \%)$.

\section{Instrumentos}

Os participantes responderam à versão castelhana do Questionário de Valores de Schwartz, QVS (Ros \& Grad, 1991), que consta dos 56 itens da versão original em Inglês (Schwartz, 1992), e de mais dois itens que foram introduzidos pelos adaptadores: trabalho e identidade nacional. Para respondê-lo, a pessoa deve indicar a importância que cada valor específico tem como um princípio-guia na sua vida. Para tanto, utiliza uma escala de resposta que varia de -1 (oposto aos meus valores) a 7 (de suprema importância). $\mathrm{O}$ QVS é dividido em duas partes, a primeira vai do item 1 ao 32, e a segunda do 33 ao 58. É solicitado ao respondente que siga esta ordem e que primeiro leia todos os itens, escolhendo o que é contrário aos seus princípios-guia, que receberá a pontuação-1; depois é solicitado a identificar o valor de suprema importância que receberá a pontuação7. Recomenda-se usar o menos possível as pontuações -1 e 7.Este mesmo procedimento é repetido para as duas listas de valores. Neste estudo, foi considerada uma versão reduzida ad hoc deste instrumento (Grad et al., 1993), que se compõe dos 37 valores específicos listados na Tabela 1.

O QVS foi validado através de uma pesquisa com mais de 40 amostras em 20 países diferentes, tendo sido observadas similaridades trans-culturais no conteúdo e na estrutura dos valores. Comprovou-se uma consistência de 44 do total de 56 valores originais, dos quais os 37 considerados foram encontrados em pelo menos $85 \%$ dos países e em $90 \%$ das amostras estudadas (Schwartz \& Sagiv, 1995). Assim, é coerente esperar uma estrutura dos valores com base neste conjunto de itens, os quais permitem representar os dez domínios motivacionais postulados pela teoria em questão.

Os participantes também foram solicitados a responder um questionário com perguntas demográficas (por exemplo, sexo, idade, religiosidade), que invariavelmente constava como a última parte do estudo.

\section{Procedimentos}

Os questionários foram aplicados coletivamente na sala de aula. Uma vez solicitada a colaboração voluntária dos potenciais participantes, os responsáveis pela coleta dos dados seguiram os seguintes passos: (a) comentavam que estava sendo realizado um estudo para conhecer as atitudes, opiniões e condutas das pessoas sobre diferentes aspectos da sua vida; (b) enfatizavam a necessidade de que as respostas fossem dadas individualmente, de acordo com o que pensava cada um, sem levar em conta o que seria mais desejável socialmente; e (c) indicavam que toda informação era confidencial, e que somente seriam tratadas estatisticamente de forma conjunta. Uma média de 20 minutos foi suficiente para responder aos questionários.

\section{Análise Estatística}

A Análise Fatorial Confirmatória foi realizada com o programa LISREL 8 (Joreskög \& Sörbom, 1989). A matriz de entrada foi a de correlações de Pearson, considerando os 37 valores antes indicados. Os parâmetros “"”' (saturações fatoriais) e "f" (correlações entre os fatores) foram livremente estimados, tendo sido adotado o estimador Quadrados Mínimos não Ponderados (ULS). Quando consideradas grandes amostras, N > 200 (Watkins, 1989), este oferece resultados bastante similares a outros estimadores, como os Quadrados Mínimos Generalizados e a Máxima Verosimilhança, porém é menos dependente do critério de distribuição multi-normal ao qual estão sujeitos estes últimos (Bollen, 1989). Este aspecto atende à natureza dos valores humanos, cuja distribuição é claramente enviesada devido ao seu componente de desejabilidade social (Schwartz, Verkasalo, Antonovsky \& Sagiv, 1997). Os seguintes índices estatísticos serão apresentados:

$\chi^{2}$ (Qui-Quadrado). Comprova a probabilidade de um modelo se ajustar aos dados. Um valor de $\chi^{2}$ estatisticamente significativo indica discrepância entre os dados e o modelo teórico sob análise.

Razão $\chi^{2}$ / gl (graus de liberdade). É considerada como uma bondade de ajuste subjetiva. Embora não exista uma valor crítico exato para decidir sobre a adequação ou não do modelo, na prática se aceitam índices que sejam iguais ou inferiores a 5,00 (Byrne, 1989).

Índice de Bondade de Ajuste (GFI). É uma medida de variabilidade explicada pelo modelo. Quando se ajusta o modelo aos graus de liberdade, correspondendo ao número de variáveis consideradas, fala-se em AGFI. Segundo Rhee, Uleman e Lee (1996), um GFI de 0,80 ou mais indica que o modelo se ajusta aos dados. Estes índices, contrariamente ao $\chi^{2}$, não são afetados pelo tamanho da amostra de participantes (Saris \& Stronkhorst, 1984).

Raiz Quadrada Média Residual (RMSR). Como seu próprio nome sugere, baseia-se diretamente nos residuais. Um valor próximo de zero significa que o modelo teórico se ajusta aos dados, isto porque os residuais se aproximam de zero (Joreskög \& Sörbom, 1989). 


\section{Resultados}

Em relação ao conteúdo dos tipos de valores, a solução com os dez fatores foi estabelecida. Na Tabela 2 são resumidos os resultados principais desta análise fatorial confirmatória. No geral, o modelo teórico se ajustou satisfa- toriamente aos dados. Embora tenha apresentado um valor de Qui-quadrado estatisticamente significativo $\left[\chi^{2}(584)=\right.$ 2294,65, $\mathfrak{p}<0,01]$, outros indicadores de bondade de ajuste dão suporte a esta apreciação: a razão $\chi^{2} / \mathrm{gl}$ foi de 3,81 , com um $G F I$ de 0,87 e o RMSR de 0,08 .

Tabela 2

Estrutura Fatorial dos Tipos Motivacionais de Valores

\begin{tabular}{|c|c|c|c|c|c|c|c|c|c|c|}
\hline \multirow[b]{2}{*}{ Item } & \multicolumn{10}{|c|}{ TIPOS MOTIVACIONAIS DE VALOR } \\
\hline & $\begin{array}{c}\mathrm{RE} \\
(0,68)\end{array}$ & $\begin{array}{c}\mathrm{HE} \\
(0,62)\end{array}$ & $\begin{array}{c}\text { TR } \\
(0,60)\end{array}$ & $\begin{array}{c}\mathrm{CO} \\
(0,55)\end{array}$ & $\begin{array}{c}\text { ES } \\
(0,76)\end{array}$ & $\begin{array}{c}\text { PO } \\
(0,67)\end{array}$ & $\begin{array}{c}\mathrm{SE} \\
(0,50)\end{array}$ & $\begin{array}{c}\mathrm{AU} \\
(0,37)\end{array}$ & $\begin{array}{c}\text { UN } \\
(0,68)\end{array}$ & $\begin{array}{c}\text { BE } \\
(0,53)\end{array}$ \\
\hline 57 & 0,64 & & & & & & & & & \\
\hline 36 & 0,61 & & & & & & & & & \\
\hline 45 & 0,58 & & & & & & & & & \\
\hline 52 & & 0,73 & & & & & & & & \\
\hline 04 & & 0,68 & & & & & & & & \\
\hline 53 & & & 0,63 & & & & & & & \\
\hline 42 & & & 0,61 & & & & & & & \\
\hline 38 & & & 0,43 & & & & & & & \\
\hline 07 & & & 0,42 & & & & & & & \\
\hline 19 & & & 0,34 & & & & & & & \\
\hline 49 & & & & 0,62 & & & & & & \\
\hline 12 & & & & 0,54 & & & & & & \\
\hline 21 & & & & 0,46 & & & & & & \\
\hline 27 & & & & & 0,77 & & & & & \\
\hline 10 & & & & & 0,67 & & & & & \\
\hline 39 & & & & & 0,61 & & & & & \\
\hline 13 & & & & & & 0,69 & & & & \\
\hline 03 & & & & & & 0,64 & & & & \\
\hline 29 & & & & & & 0,56 & & & & \\
\hline 09 & & & & & & & 0,51 & & & \\
\hline 14 & & & & & & & 0,49 & & & \\
\hline 23 & & & & & & & 0,45 & & & \\
\hline 17 & & & & & & & & 0,53 & & \\
\hline 05 & & & & & & & & 0,42 & & \\
\hline 33 & & & & & & & & 0,29 & & \\
\hline 32 & & & & & & & & & 0,64 & \\
\hline 18 & & & & & & & & & 0,58 & \\
\hline 01 & & & & & & & & & 0,57 & \\
\hline 40 & & & & & & & & & 0,53 & \\
\hline 31 & & & & & & & & & 0,44 & \\
\hline 37 & & & & & & & & & 0,40 & \\
\hline 28 & & & & & & & & & 0,39 & \\
\hline 30 & & & & & & & & & 0,23 & \\
\hline 51 & & & & & & & & & & 0,69 \\
\hline 47 & & & & & & & & & & 0,47 \\
\hline 56 & & & & & & & & & & 0,42 \\
\hline 11 & & & & & & & & & & 0,34 \\
\hline
\end{tabular}

Nota: Entre parênteses figura o Alfa de Cronbach de cada tipo de valor. Todos os pesos fatoriais são diferentes de $0(\underline{t}>1,96, \underline{p}<0,05)$. Identificação dos tipos motivacionais de valores: $\mathrm{UN}=$ Universalismo, $\mathrm{BE}=$ Benevolência, $\mathrm{CO}=$ 
Como é possível observar na tabela acima, todas as cargas (saturações) fatoriais são estatisticamente diferentes de zero $(\mathrm{t}>1,96, \mathrm{p}<0,05)$, estando no intervalo de 0,23 (correspondente ao valor "amizade verdadeira" que figura em "universalismo") e 0,77 (identificado como "uma vida variada", pertencente a "estimulação"). Todas as cargas fatoriais são positivas, corroborando a adequação da distribuição do conjunto de valores específicos segundo os tipos de valores propostos pela teoria. Ainda em relação a estes tipos de valores, nesta mesma tabela também são oferecidos os seus índices de consistência interna (Alfa de Cronbach, $\alpha$ ), os quais variaram de 0,37 (autodireção) a 0,76 (estimulação), com um a médio de 0,60 .

No que diz respeito a estrutura de compatibilidade e conflito dos tipos motivacionais, segundo estabelece o modelo de Schwartz, na Tabela 3 é apresentada a matriz de correlações $(\phi)$ entre os fatores resultantes da análise prévia. Vale ressaltar que, diferentemente da análise de escalonamento multidimensional que define a compatibilidade e o conflito através da proximidade e afastamento dos tipos de valores, respectivamente, na análise fatorial confirmatória estas podem ser avaliadas através da magnitude e da direção das correlações entre os fatores. Uma correlação positiva e significativa denota compatibilidade entre um par de tipos motivacionais de valores, enquanto que a negativa e significativa seria indício de conflito. Uma correlação neutra ou a ortogonalidade de dois tipos de valores carece de significado neste contexto, isto é, não tem sido prevista pela teoria.

Como é possível perceber na Tabela 3, são corroboradas todas as compatibilidades entre os tipos de valores. Estas estão localizadas principalmente na diagonal da matriz de correlações; a menor corresponde aos tipos de valores "hedonismo" e "realização" $(\phi=0,26, p<0,05)$ e a maior à "segurança" e "conformidade" $(\phi=0,90, p<0,05)$. As demais compatibilidades previstas eram: "benevolência" e "universalismo" $(\phi=0,84, p<0,05)$, "benevolência" e "conformidade" $(\phi=0,66, p<0,05)$, "conformidade" e "tradição" $(\phi=0,74, p<0,05)$, "tradição" e "segurança" $(\phi=$ $0,70, p<0,05)$, "segurança” e "poder" $(\phi=0,55, p<0,05)$, "poder" e "realização" $(\phi=0,61, p<0,05)$, "hedonismo" e "estimulação" $(\phi=0,59, p<0,05)$, "estimulação" e "autodireção" $(\phi=0,71, p<0,05)$, e “autodireção" e "universalismo" $(\phi=0,52, p<0,05)$.

No que se refere aos conflitos, os resultados não suportaram plenamente a teoria. Os tipos motivacionais "realização" e "benevolência" se correlacionaram direta em lugar de inversamente $(\phi=0,19, p<0,05)$, e alguns dos pares de tipos motivacionais se revelaram ortogonais ao invés de contrários, a saber: "conformidade" e "autodireção" ( $\phi=-0,07$, $p>0,05$ ), "conformidade" e "estimulação" ( $\mathrm{f}=0,04, p>$ $0,05)$, "conformidade" e "hedonismo" $(\phi=-0,04, p>0,05)$, “estimulação" e "tradição" $(\phi=0,02, p>0,05)$, "estimulação" e "segurança" $(\phi=0,06, p>0,05)$, e

Tabela 3.

Identificação de compatibilidades e conflitos dos tipos motivacionais de valores através da matriz $\phi$

\begin{tabular}{|c|c|c|c|c|c|c|c|c|c|}
\hline $\mathrm{BE}$ & $0,84 *$ & & & & & & & & \\
\hline $\mathrm{CO}$ & $0,19 *$ & $0,66^{*}$ & & & & & & & \\
\hline $\mathrm{TR}$ & $0,31 *$ & $0,68 *$ & $0,74 *$ & & & & & & \\
\hline $\mathrm{SE}$ & $0,23 *$ & $0,38^{*}$ & $0,90^{*}$ & $0,70^{*}$ & & & & & \\
\hline $\mathrm{PO}$ & $-0,33^{*}$ & $-0,40^{*}$ & $0,27 *$ & $-0,03$ & $0,55^{*}$ & & & & \\
\hline $\mathrm{RE}$ & 0,03 & $0,19 *$ & $0,56^{*}$ & $0,11^{*}$ & $0,66^{*}$ & $0,61^{*}$ & & & \\
\hline $\mathrm{HE}$ & $0,15^{*}$ & $-0,11^{*}$ & $-0,04$ & $-0,10^{*}$ & $0,15^{*}$ & $0,35^{*}$ & $0,26^{*}$ & & \\
\hline ES & $0,31 *$ & 0,10 & 0,04 & 0,02 & 0,06 & $0,19^{*}$ & $0,29 *$ & $0,59^{*}$ & \\
\hline \multirow[t]{2}{*}{$\mathrm{AD}$} & $0,52 *$ & 0,29 & $-0,07$ & $-0,13^{*}$ & $-0,20^{*}$ & $-0,11 *$ & $0,23 *$ & $0,48^{*}$ & $0,71 *$ \\
\hline & UN & $\mathrm{BE}$ & $\mathrm{CO}$ & TR & SE & $\mathrm{PO}$ & $\mathrm{RE}$ & $\mathrm{HE}$ & ES \\
\hline
\end{tabular}

Nota: * Correlação $(\phi)$ entre os tipos de valores diferente de $0(\mathrm{t}>1,96, \underline{\mathrm{p}}<0,05)$. Identificação dos tipos de valores: $\mathrm{UN}=$ Universalismo, $\mathrm{BE}=$ Benevolência, $\mathrm{CO}=$ Conformidade, $\mathrm{TR}=$ Tradição, $\mathrm{SE}=$ Segurança, $\mathrm{PO}=$ Poder, $\mathrm{RE}=$ Realização, HE $=$ Hedonismo, $\mathrm{ES}=$ Estimulação, e AD = Autodireção. 
"universalismo" e "realização" $(\phi=0,03, p>0,05)$. Os seguintes tipos de valores apresentaram um padrão de correlação dentro do que seria esperado: "universalismo" e "poder" $(\phi=-0,33, p<0,05), 0$ "poder" e "benevolência” $(\phi=$ $-0,40, p<0,05)$, "tradição" e "autodireção" $(\phi=-0,13, p<$ $0,05)$, "autodireção" e "segurança" $(\phi=-0,20, p<0,05) \mathrm{e}$ "hedonismo" e "tradição" $(\phi=-0,10, p<0,05)$.

\section{Discussão}

Relembrando, o propósito deste artigo foi comprovar dois dos aspectos centrais da teoria dos valores humanos proposta por Schwartz (1992, 1994): (1) o conteúdo dos tipos motivacionais de valores, e (2) suas relações de compatibilidade e conflito entre si. Para tanto, considerou-se uma amostra ampla de participantes $(\mathrm{N}=477)$, embora limitada a dois cursos universitários. Assumindo esta limitação, espera-se que tenham sido reunidas provas que, somadas às existentes na literatura (por exemplo, Ros \& Grad, 1991; Schwartz \& Sagiv, 1995; Tamayo \& Schwartz, 1993), permitam dirimir as dúvidas apontadas por Tamayo (1997) quanto à adequação desta teoria.

$\mathrm{O}$ fato do $\chi^{2}$ ter apresentado um valor significativo não constitui um problema neste contexto. Identificada por Joreskög e Sörbom (1989) como um índice de "maldade de ajuste", esta estatística apresenta sérias limitações quando o critério de multinormalidade não é satisfeito, como ocorre quando se tratam de variáveis como os valores humanos. Neste sentido, recomenda-se que o mesmo seja deixado de lado em detrimento de outros indicadores de bondade de ajuste, a exemplo da razão $\chi^{2} / \mathrm{gl}$ e do GFI (Byrne, 1989). Seguindo estas sugestões, é possível interpretar o modelo de Schwartz como sendo satisfatório; embora a solução com dez tipos de valores não apresente índices de ajuste excepcionais (Van de Vijver \& Leung, 1997), está dentro do que tem sido recomendado, como se descreve na sessão de análise estatística.

Os índices de consistência interna ( $\alpha$ de Cronbach) encontrados para cada tipo motivacional também reforçam a adequação destes. Considerando a média geral $(\alpha=0,60)$, percebe-se que está dentro do que tem sido encontrado em outros estudos: 0,56 (Sagiv \& Schwartz, 1995) a 0,69 (Feather, 1996). Os tipos de valores "autodireção" e "estimulação", que no presente estudo obtiveram o menor ( $\alpha=0,37)$ e o maior $(\alpha=0,76)$ índice de consistência interna, respectivamente, também o fizeram na pesquisa de Feather (1996): $\alpha=0,58$ e 0,79 , respectivamente. Vale ressaltar que neste último estudo foram considerados todos os valores específicos que teoricamente pertencem aos tipos de valores em discussão (Schwartz, 1992), o que normalmente resultaria em maiores $\alpha$ do que os obtidos no presente estudo, que tratou com uma amostra reduzida de valores específicos (ver Tabela 1).
A evidência a favor da estrutura com os dez tipos de valores não significa que a teoria em questão seja plenamente satisfatória, considerando-se o tratamento estatístico que foi efetuado. Enquanto foram corroboradas todas as compatibilidades entre os tipos de valores previstas pela teoria, unicamente cinco pares de doze conflitos hipotéticos apresentaram correlações na direção esperada. Esta situação parece sugerir ao menos duas possibilidades: (a) reflete o contexto cultural em que a pesquisa foi realizada; em países ibero-americanos, como no caso específico da Espanha ou mesmo no que se refere ao Brasil, existe uma mistura de identidades e uma combinação de estilos de pensamentos e de condutas que faz possível a convivência de orientações valorativas em princípio opostas (ver também Gouveia, 1998; Ros \& Gómez, 1997; Tamayo, 1997); e (b) acentua a própria natureza dos valores, considerados como princípios-guia desejáveis pessoal e socialmente (Schwartz et al., 1997), o que torna mais prováveis que estejam diretamente relacionados entre si (Gouveia, 1998). Esta concepção não nega o conflito de valores, senão que afirma que este é menos evidente do que a compatibilidade (Gouveia, 2000).

A respeito do parágrafo anterior, deve-se acrescentar que a noção de conflito como oposição, expressa através do coeficiente de correlação negativo e estatisticamente significativo, pode ser elementar neste contexto. Segundo a teoria de Schwartz e seus colaboradores (Schwartz, 1992, 1994; Schwartz \& Bilsky, 1987, 1990; Tamayo \& Schwartz, 1993), os tipos de valores conformam uma estrutura circular, sendo compatíveis os que estão em regiões adjacentes (por exemplo, "conformidade" e "tradição") e conflituosos aqueles que figuram em regiões opostas (por exemplo, "benevolência" e "poder") (ver Figura 1). Não obstante, vale lembrar que na SSA, tais tipos de valores não correspondem a eixos ou fatores, como na análise fatorial, mas sim a regiões que correspondem a determinados conjuntos de valores específicos no espaço bidimensional. Visualizar uma região em oposição a outra não significa que se levem em conta as especificidades dos valores que as compõem. No caso da análise fatorial confirmatória, tais valores são somados para formar cada tipo de valor, podendo ser minimizadas e/ou neutralizadas as dispersões, o que poderia resultar em uma correlação próxima a zero entre dois tipos de valores em princípio conflituosos. Deste modo, tal correlação pode vir a ser erroneamente interpretada como ausência de "conflito".

Finalmente, Tamayo (1997) sugere utilizar modelos de equações estruturais, a exemplo da análise fatorial confirmatória, para comprovar a teoria dos tipos de valores de Schwartz (1992). Tem razão no que se refere à sua objetividade e, provavelmente, pensou também na vantagem desta técnica contar com critérios específicos e precisos para decidir sobre o ajuste de um modelo aos dados empíricos 
(por exemplo, $\chi^{2}, \mathrm{GFI}$ ). Existem também vantagens quando comparada com outras técnicas. Por exemplo, a análise fatorial exploratória produz um fator geral que concentra muitos dos itens de um instrumento (Davison \& Skay, 1991), impedindo cotejar a natureza complexa de algumas tipologias. O escalonamento multidimensional é menos susceptível ao problema do fator comum, porém trabalha com dimensões em lugar de eixos, sugerindo um procedimento subjetivo no momento de comprovar uma teoria (Shye, Elizur \& Hoffman, 1994). Apesar destes aspectos positivos, percebe-se claramente que a análise fatorial confirmatória não consegue apreender a natureza dinâmica da estrutura de compatibilidade e conflito dos tipos de valores proposta pela teoria em questão, cuja adequação tem sido demonstrada tanto internamente (Gouveia, 2000; Ros \& Grad, 1991; Tamayo \& Schwartz, 1993) como em relação a critérios externos, a exemplo da religiosidade (Bilsky \& Peters, 1999; Gouveia, Clemente \& Vidal, 1998; Schwartz \& Huismans, 1995).

\section{Conclusão}

Apesar das críticas que vem recebendo (Gouveia, 1998; Molpeceres, 1994), a teoria de Schwartz (1992, 1994) segue dando origem a novos estudos. É provavelmente a teoria dos valores humanos mais conhecida atualmente na Psicologia, e conta com o mérito (ou a comodidade) de ter sido criada a partir do embasamento proposto por Rokeach (1973). Conseguiu resolver antigas limitações deste modelo, como a falta de uma estrutura delimitada e a imposição de ordenação de prioridades dos valores (Tamayo, 1997), e parte atualmente para a elaboração de instrumentos de medida dos valores mais curtos e dirigidos a determinados grupos, como os adolescentes. Certamente esta teoria tem muito que oferecer, porém são necessárias novas incursões que resolvam as limitações da área, que não são exclusivamente suas. Bond (1988), desde uma perspectiva mais oriental, oferece uma possibilidade, mostrando que existem valores que geralmente não têm sido contemplados em outras tipologias (por exemplo, "ter poucos desejos", "constância pessoal").

Embora Schwartz tenha dado a possibilidade de serem incluídos valores específicos das culturas participantes do seu projeto de pesquisa (Tamayo, 1997), alguns valores seguem sem aparecer. Se se assume que os valores são representações das necessidades (Rokeach, 1973; Schwartz \& Bilsky, 1987), as mais básicas, como as de alimento e água, deveriam figurar de alguma forma. Por exemplo, Pepper (1958) sugere um valor denominado de "sobrevivência", que pouco aparece na literatura, talvez por este não ser relevante nas culturas onde as teorias têm surgido. A propósito, Gouveia (1998) considera este valor dentro de um grupo de valores denominados de "existência", sugerindo sua adequação para explicar o tipo de orientação denominado de "protoindividualista" (põe ênfase no atributo batalhador, que busca sua própria existência orgânica). É importante assinalar, não obstante, que toda inclusão de um novo valor deverá ser justificada teoricamente.

Em resumo, a teoria de Schwartz é, no geral, bastante adequada, mesmo quando empregam técnicas estatísticas baseadas em equações estruturais. O que não foi possível comprovar, no caso o conflito dos valores, pode-se atribuir à natureza do objeto de estudo. A estrutura dinâmica dos valores pode ser apreciada claramente através do escalonamento multidimensional, e tem demonstrado valor preditivo em relação a variáveis externas ao modelo, como o contato social com exogrupos (Sagiv \& Schwartz, 1995). Neste contexto, condicionar sua adequação às estatísticas baseadas no LISREL pode não ser muito producente.

\section{Agradecimentos}

A preparação deste artigo foi financiada em parte pelo CNPq, através de uma bolsa de Produtividade em Pesquisa concedida ao primeiro autor. Os autores agradecem a esta instituição.

\section{Referências}

Bilsky, W., \& Peters, M. (1999). Estructura de los valores y la religiosidad. Una investigación comparada realizada en México. Revista Mexicana de Psicología, 16, 77-88.

Bisquerra, R. (1989). Introducción conceptual al análisis multivariable: Un enfoque con los paquetes estadísticos SPSS-X, BMDP, LISREL y $S P A D$. Barcelona: PPU.

Bollen, K. A. (1989). Structural equations with latent variables. New York: John Wiley \& Sons.

Bond, M. H. (1988). Finding universal dimensions of individual variation in multicultural studies of values: The Rokeach and Chinese Value Surveys. Journal of Personality and Social Psychology, 55, 1009-1015.

Braithwaite, V., \& Law, H. G. (1985). Structure of human values: Testing the adequacy of the Rokeach Value Survey. Journal of Personality and Social Psychology, 49, 250-263.

Byrne, B. M. (1989). A primer of LISREL: Basic applications and programming for confirmatory factor analytic models. New York: Springer-Verlag.

Davison, M. L. \& Skay, C. L. (1991). Multidimensional scaling and factor models of test and item responses. Psychological Bulletin, 110, 551-556.

Feather, N. T. (1984). Protestant ethic, conservatism, and values. Journal of Personality and Social Psychology, 46, 1132-1141.

Feather, N. T. (1996). Reactions to penalties for an offense in relation to authoritarianism, values, perceived responsability, perceived seriousness, and deservingness. Journal of Personality and Social Psychology, 71, 571-587.

Gendre, F., \& Dupont, H.B. (1992). Structure of the Schwartz questionnaire for values. Revue Suisse de Psychologie, 51, 128-134.

Grad, H., Ros, M., Álvaro, J. L., \& Torregrosa, J. R. (1993). Influencias de factores universales, culturales y ocupacionales en el sistema personal de valores en España. Interacción Social, 3, 181-199. 
Gouveia, V. V. (1998). La naturaleza de los valores descriptores del individualismo y del colectivismo: Una comparación intra e intercultural. Tese de Doutorado não-publicada. Faculdade de Psicologia, Universidade Complutense de Madri, Espanha.

Gouveia, V. V. (2000). El conflicto de valores en las culturas iberoamericanas: Consideraciones acerca de Brasil y España. In D. Caballero, M. T. Méndez \& J. Pastor (Orgs.), La mirada psicosociológica: Grupos, procesos, lenguages y culturas (pp. 805810). Madrid: Biblioteca Nueva.

Gouveia, V. V., Clemente, M., \& Vidal, M. A. (1998). El Cuestionario de Valores de Schwartz (CVS): Propuesta de adaptación en el formato de respuesta. Revista de Psicología Social, 13, 463-469.

Günther, H. (1981). Uma tentativa de traduzir e adaptar a Escala de Valores de Rokeach para uso no Brasil. Arquivos Brasileiros de Psicologia, 33, 58-72.

Joreskög, K. G., \& Sörbom, D. (1989). LISREL 7 user's reference guide. Mooresville: Scientific Software.

Karp, D. G. (1996). Value and their effect on pro-environment behavior. Environment and Behavior, 28, 111-133.

Menezes, I., \& Campos, B. P. (1997). The process of value-meaning construction: a cross-sectional study. European Journal of Social Psychology, 27, 55-73.

Molpeceres, M. A. (1994). El sistema de valores: su configuración cultural y su socialización familiar en la adolescencia. Tese de Doutorado nãopublicada. Faculdade de Psicologia, Universidade de Valência, Espanha.

Oishi, S., Schimmack, U., Diener, E., \& Suh, E.M. (1998). The measurement of values and individualism and collectivism. Personality and Social Psychology Bulletin, 24, 1177-1189.

Pepper, S. C. (1958). The sources of values. Berkeley: University of California Press.

Rhee, E., Uleman, J. S., \& Lee, H. K. (1996). Variations in collectivism and individualism by ingroup and culture: Confirmatory factor analysis. Journal of Personality and Social Psychology, 71, 1037-1054.

Rokeach, M. (1973). The nature of human values. New York: Free Press.

Ros, M., \& Gómez, A. (1997). Valores personales individualistas y colectivistas y su relación con la autoestima colectiva. Revista de Psicología Social, 12, 179-198.

Ros, M., \& Grad, H. (1991). El significado del valor trabajo como relacionado a la experiencia ocupacional: Una comparación de profesores de EGB y estudiantes del CAP. Revista de Psicología Social, 6, 181-208.

Sagiv, L., \& Schwartz, S. H. (1995). Value priorities and readiness for outgroup social contact. Journal of Personality and Social Psychology, $69,437-448$.
Saris, W., \& Stronkhorst, H. (1984). Causal modelling in nonexperimental research. Amsterdam: Sociometric Research Foundation.

Schwartz, S. H. (1990). Individualism-collectivism: Critique and proposed refinements. Journal of Cross-Cultural Psychology, 21, 139-157.

Schwartz, S. H. (1992). Universals in the context and structure of values: theoretical advances and empirical tests in 20 countries. In M. Zanna (Org.), Advances in experimental social psychology (vol. 25, pp. 165). Orlando: Academic Press.

Schwartz, S. H. (1994). Are there universal aspects in the structure and contents of human values? Journal of Social Issues, 50, 19-45.

Schwartz, S. H., \& Bilsky, W. (1987). Toward a universal psychological structure of human values. Journal of Personality and Social Psychology, 53, 550-562.

Schwartz, S. H., \& Bilsky, W. (1990). Toward a theory of the universal content and structure of values: Extensions and cross-cultural replications. Journal of Personality and Social Psychology, 58, 878- 891.

Schwartz, S. H., \& Huismans, S. (1995). Value priorities and religiousity in four western religions. Social Psychology Quarterly, 58, 88-107.

Schwartz, S. H., \& Sagiv, L. (1995). Identifying culture-specifics in the content and structure of values. Journal of Cross-Cultural Psychology, 26, 92-116.

Schwartz, S. H., Verkasalo, M., Antonovsky, A., \& Sagiv, L. (1997). Value priorities and social desirability: Much substance, some style. British Journal of Social Psychology, 36, 3-18.

Shye, S., Elizur, D., \& Hoffman, M. (1994). Introduction to facet theory: Content design and intrinsic data analysis in behavioral research. Thousand Oaks: Sage.

Tamayo, A. (1988). Influência do sexo e da idade sobre o sistema de valores. Arquivos Brasileiros de Psicologia, 38, 91-104.

Tamayo, A. (1994). Hierarquia de valores transculturais e brasileiros. Psicologia: Teoria e Pesquisa, 10, 269-285.

Tamayo, A. (1997). Os valores do brasileiro: uma década de pesquisa. Cadernos de Psicologia, 1, 115-134.

Tamayo, A. \& Schwartz, S.H. (1993). Estrutura motivacional dos valores humanos. Psicologia: Teoria e Pesquisa, 9, 329-348.

Thomas, W. I., \& Znaniecki, F. (1918). The Polish peasant in Europe and America. Boston: University of Chicago Press.

Van de Vijver, F. J. R., \& Leung, K. (1997). Methods and data analysis for cross-cultural reserach. Newbury Park: Sage.

Vera, J. J., \& Martínez, M. del C. (1994). Preferencias de valores en relación con los prejuicios hacia endogrupos. Anales de Psicología, 10, 29-40.

Watkins, D. (1989). The role of confirmatory factor analysis in cross-cultural research. International Journal of Psychology, 24, 685-701.

Valdiney V. Gouveia, doutor em Psicologia pela Universidad Complutense de Madrid, Espanha, é professor do Departamento de Psicologia da Universidade Federal da Paraíba. Endereço para correspondência: Universidade Federal da Paraíba, CCHLA - Departamento de Psicologia, 58059-900, João Pessoa, PB.E-mail: vgouveia@cchla.ufpb.brou vvgouveia@uol.com.br.

Eva Martínez é doutoranda em Psicologia Social, Departamento de Psicologia Social, Facultad de Sociología, Universidad Complutense de Madrid, Espanha. E-mail:emartinez@ pharma-consult.com Maja Meira é graduada em Psicologia pela Universidade Federal da Paraíba (PB). E-mail: maja_meira@uol.com.br.

Taciano Lemos Milfont é mestre em Psicologia Social pela Universidade Federal da Paraíba. Email:milfont@hotmail.com. 